\title{
Fetal parvovirus syndrome
}

INSERM

\section{Source}

INSERM. (1999). Orphanet: an online rare disease and orphan drug data base. Fetal parvovirus syndrome. ORPHA:295

Foetal parvovirus syndrome is a foetopathy likely to occur when a pregnant woman is infected by parvovirus B19. In adults, the virus causes a butterfly erythema infectiosum (also called Fifth Disease; 'slapped cheek disease') and flu-like symptoms with symmetric polyarthralgias, which usually do not warrant prenatal diagnosis. 